\title{
Day-time effect on postural stability in young sportsmen
}

\author{
Luca Russo ${ }^{1}$ \\ Umberto D'Eramo \\ Johnny Padulo 3,4 \\ Calogero Foti 5 \\ Riccardo Schiffer 2 \\ Fabio Scoppa ${ }^{2}$
}

1 Department of Applied Clinical Sciences and Biotechnologies, University of L'Aquila, Italy

2 Faculty of Medicine and Dental Surgery, Sapienza University of Rome, Italy

3 University eCampus, Novedrate, Italy

4 Tunisian Research Laboratory "Sports Performance Optimization" National Center of Medicine and Science in Sport, Tunis, Tunisia

5 Tor Vergata University, Rome, Italy

Corresponding author:

Johnny Padulo

University eCampus

Via Isimbardi, 10

22060 Novedrate, Italy

E-mail: sportcinetic@gmail.com

\section{Summary}

Background: stabilometry is commonly used from clinicians and posturologists for stability and postural assessment of patients. In the daily practice a large number of posturologists are usual to control the effect of their treatments on the same subject through stabilometry but tests are not always performed at the same time, so it is necessary to be sure that data are not influenced by the time-of-day. The aim of this study was to evaluate the time-of-day effect on stabilometric parameters and theirs variation.

Methods: for this aim 61 healty sportsmen performed on four repetitions of the stabilometric test during the same day (morning - evening). Data collected from each test were: centre of pressure sway path length, Naiperian logarithm of the ellipse of confidence area, of the $x$ mean and of the $y$ mean, eccentricity of the ellipse area.

Results: no significant difference was found in any parameter at any time of the day. Intra-class Correlation Coefficient value confirmed the reliability of the stabilometric evaluation in healty sub- jects (sway path length 0.836 and 0.816 for open and closed eyes, respectively).

Conclusion: these findings suggest that stabilometry evaluation is not affected by a time-of-day, therefore the test and retest in different time of day is reliable to assess postural sway.

KEY WORDS: balance, circadian variation, intra/inter variability, reliability, testing, stabilometry, time of day.

\section{Introduction}

Stabilometry is widely used to study the orthostatic postural control in sport science ${ }^{1,2}$ and clinical daily practice ${ }^{3,4}$. Maintaining balance during the natural orthostasis is a complex task for humans because of the intrinsic instability of the position ${ }^{5}$; stabilometry does not measure balance but the stability properties of the human system while keeping a balanced standig position, because balance does not correspond to an absolute reality but it is a position-specific skill. Individuals keep the orthostatic position bringing forward the mechanic perturbations thanks to postural adjustements that minimize instability ${ }^{6}$. Stabilometry studies this aspects both for healthy and unhealthy people or in particular cases of life $e^{7-11}$.

It is well known and certain that circadian rhythms ${ }^{12}$, 13 strongly influenced the everyday life of individuals and during the whole day different hormonal profiles are present, having an effect on the wake-sleep cy$\mathrm{cle}^{14}$. For this reason it can be supposed that the hormonal profile at the moment of the postural evaluation with stabilometry could significantly affect the results. This hypothesis means that performing two stabilometric test at different time of the day could make it impossible to compare the clinical results and could limit the interpretation of the effects of a postural treatment on the same subject ${ }^{15-20}$.

The aim of this study is to test the hypothesis that circadian rhythms ${ }^{13}$ can affect the postural stability in a sample of healthy male subjects through four repeated and constant measures during the day. Other researchers have recentely studied this phenomenon ${ }^{21}$, 22 , two study confirmed the lack of a time-of-day effect on stabilometric data ${ }^{21,23}$ and one study found a difference in stabilometric data during the day 22 . The number of measurements during the same day, the number and gender of subjects and the used protocol, except one study ${ }^{23}$, were different compared to this study; for these reason in literature there is a lack 
of clarity about the theme and further study are needed. The present study differs from other authors' ones for only-male gender, larger sample size and more measurements.

\section{Materials and methods}

Sixty-one young healthy male volunteers (age $22 \pm 3.4$ yrs, body height $178 \pm 8.3 \mathrm{~cm}$, body mass $77 \pm 16.4 \mathrm{~kg}$ ) participated to this study. Subjects were recruited from the Sports Science Faculty of L'Aquila volunteer list. Each subject was fully informed and trained about the test's procedures and everyone gave the written informed approval to partecipate to the study in according with the guideline of the Muscle, Ligament and Tendons Journal ${ }^{24}$.

Participants were selected according to the following criteria of inclusion:

1. Age between 18 and 30 years $^{25,26}$

2. No pain symptoms referred to the postural system or the muscle-skeletal apparatus ${ }^{27}$

3. No chronic pathology26

4. No medicine assumption

5. No alcohol or drug assumption 26

6. No night shift worker

7. No sleep disorder

8. No neurological disorder 23,27

9. No vestibular disorder or injury or surgey in the last 12 months before the test ${ }^{21}$.

Due to the chronobiological nature of the study, authors chose to study only male subjects because of the impossibility to exactly control the female menstrual cycle.

\section{Procedures}

Stabilometric tests were performed on a platform (FreeMed, Sensor Medica, Guidonia, Italy) ${ }^{28}$. A stabilometric laboratory corresponding to the indications of the International Society do Posturology was chosen for the tests ${ }^{29}$. Each subject was tested four times during the same day: first test between 9 a.m. and 11 a.m., second test between 11 (10') a.m. and 1 (10') p.m., third test between 3 p.m. and 5 p.m., fourth test between 5 (10') p.m. and 7 (10') p.m. No measurement was performed during the night because the absence of postural reflexes during sleep. A maximum of 13 subjects could be tested at each time range; tests lasted 5 days in order to collect the whole sample of participants.

\section{Subjects had to follow some indications for the tests procedures:}

1. Punctuality

2. Silence and concentration during the test

3. Prohibition to get drugs, to smoke and to drink alcohol, coffee or exciting drinks during the whole day of the test
4. Prohibition to eat chewing gum before the test

5. To sleep at least 7 hours the night before the test and to wake up at least 2 hours before the first morning test

6. To keep the same clothes for the testing sessions. Before each test everyone had to leave every kind of metal wearing, to avoid possible micro galvanism ${ }^{30}$, after this operation the tested subject waited 3-min sitting down and then performed the stabilometric test both keeping his eyes open and closed. Stabilometric tests respected the international standardization criteria $^{29}$ : visual target within 5 meters, head in neutral position, silence, first acquisition with open and then with closed eyes, each test was 51.2-sec, feet were positioned with heel separated of $2-\mathrm{cm}$ and the feet tips $30^{\circ}$ wide apart, arms were close fitted to thighs. Authors chose to use the same protocol of Gagey ${ }^{23}$ to be able to compare the results of the study, despite new indications for the standardization of the clinical stabilometric test have been recently provid$\mathrm{ed}^{31}$. Instructions before each test were always the same to avoid possible changes on stabilometry ${ }^{32}$.

\section{Data collection}

Length of centre of pressure (Cop) sway path $(\mathrm{CSPL})^{31}$, Naiperian logarithm of the ellipse of confidence area (LNEA), of the $x$ mean (LNX) and of the $y$ mean $(L N Y)^{23}$, eccentricity of the ellipse area (EEA). The transformation of some data in Naiperian logarithm aimed at normalizing this non-linear data ${ }^{23}$. Open eyes and closed eyes conditions were took in consideration for data analysis.

\section{Statistical analysis}

Statistical analyses were performed with SPSS 16.0 (SPSS Inc. Chicago, IL). The normally distribution of the population was tested with Shapiro-Wilk test and the homogeneity of variances was verified with the Bartlett's test. Post-hoc power analysis was conducted with the program $\mathrm{G}^{*} \mathrm{Power}^{33}$. ANOVA design with a Sidak correction and an Intra-class Correlation Coefficient (ICC) were used to evaluate differences between repetead measures during the day and to evaluate the reliability of the measures ${ }^{34}$. The level of significance was set to $p<0.05$.

\section{Results}

Stabilometric data from an amount of 488 tests are showed in Table 1 and 2. ANOVA does not show significant differences between the four series of test for any of the measured parameters both for the open eyes and the closed eyes conditions. Only CSPL and EEA show "p" values on the edge of significance, but the post-hoc comparisons underline the lack of significant variations. The CSPL shows an increasing, but not significant, trend during the day suggesting an increase in 
the energetic consumption to maintain the orthostatic position. The LNEA remains constant during the four trials; the real values of the ellipse of confidence are contained within the normal range suggested from literature $^{31}$. The center of Cop trajectory, described by the $x$ and $y$ mean values, show very little variations during the day both in the frontal and sagittal plane. The EEA, clinically referred to as the preferential direction of oscillation, shows a not significant decrease after the morning and then a plateau in the evening.

Finally the radial movement frequencies of the Cop indicate the postural strategy adopted by participants: low frequencies indicate slow postural movements probably managed by feed forward regulatory mechanisms, while high frequencies indicate fast postural adjustments probably managed by feedback regulatory mechanisms. The trend of these values show a moderate and not significant increase during the day. Reliability procedures have been made on the data for average and for single measures (Tab. 3). Both average and single measures seem to be significantly reliable, but single measures show smaller ICC values than the average ones and single measures of EEA are not significantly reliable.

Table 1. Variations of Open Eyes on stabilometric device in different time of day.

\begin{tabular}{llllll}
\hline Variable & Morning $(\mathbf{A})$ & Morning (B) & Evening (A) & Evening (B) & p value \\
\hline CSPL $(\mathbf{m m})$ & $312.52(102.80)$ & $329.41(101.15)$ & $346.95(97.88)$ & $345.48(94.00)$ & 0.189 \\
LNEA & $4.47(1.08)$ & $4.54(0.97)$ & $4.67(0.95)$ & $4.46(1.08)$ & 0.630 \\
LNX & $-1.65(0.56)$ & $-1.55(0.51)$ & $-1.53(0.58)$ & $-1.60(0.59)$ & 0.637 \\
LNY & $-1.39(0.69)$ & $-1.40(0.61)$ & $-1.36(0.55)$ & $-1.47(0.62)$ & 0.773 \\
EEA & $0.82(0.16)$ & $0.80(0.16)$ & $0.80(0.16)$ & $0.74(0.22)$ & 0.077 \\
\hline
\end{tabular}

The value are expressed as mean and standard deviation for centre of pressure of sway path length (CSPL), Naiperian logarithm of the ellipse of confidence area (LNEA), of the X mean (LNX) and of the $Y$ mean (LNY), eccentricity of the ellipse area (EEA). ANOVA with "p" value expressed within time-of-day effects: 9/11 a.m. (Morning A), 11 a.m./1 p.m. (Morning B), $3 / 5$ p.m. (Evening A), 5/7 p.m. (Evening B).

Table 2. Variations of Closed Eyes on stabilometric device in different time of day.

\begin{tabular}{llllll}
\hline Variable & Morning (A) & Morning (B) & Evening (A) & Evening (B) & p value \\
\hline CSPL $(\mathbf{m m})$ & $340.61(105.63)$ & $380.07(117.02)$ & $382.44(118.74)$ & $393.43(106.91)$ & 0.054 \\
LNEA & $3.89(1.11)$ & $4.13(1.08)$ & $3.90(1.10)$ & $4.12(1.04)$ & 0.455 \\
LNX & $-1.95(0.57)$ & $-1.76(0.61)$ & $-1.86(0.56)$ & $-1.76(0.62)$ & 0.244 \\
LNY & $-1.70(0.64)$ & $-1.63(0.63)$ & $-1.75(0.57)$ & $-1.68(0.58)$ & 0.733 \\
EEA & $0.75(0.20)$ & $0.79(0.15)$ & $0.70(0.22)$ & $0.72(0.18)$ & 0.055 \\
\hline
\end{tabular}

The value are expressed as mean and standard deviation for centre of pressure of sway path length (CSPL), Naiperian logarithm of the ellipse of confidence area (LNEA), of the X mean (LNX) and of the $Y$ mean (LNY), eccentricity of the ellipse area (EEA). ANOVA with "p" value expressed within time-of-day effects: 9/11 a.m. (Morning A), 11 a.m./1 p.m. (Morning B), $3 / 5$ p.m. (Evening A), 5/7 p.m. (Evening B).

Table 3. Single and average ICC values of Open and Closed Eyes.

\begin{tabular}{lllllllll}
\hline Variable & \multicolumn{3}{l}{ ICC Single measures } & \multicolumn{2}{c}{ "p” value } & \multicolumn{2}{c}{ ICC Average measures } & \multicolumn{2}{c}{ "p” value } \\
& OE & CE & OE & CE & OE & CE & OE & CE \\
\hline CSPL $(\mathrm{mm})$ & 0.561 & 0.526 & 0.000 & 0.000 & 0.836 & 0.816 & 0.000 & 0.000 \\
LNEA & 0.620 & 0.499 & 0.000 & 0.000 & 0.867 & 0.800 & 0.000 & 0.000 \\
LNX & 0.447 & 0.470 & 0.000 & 0.000 & 0.764 & 0.780 & 0.000 & 0.000 \\
LNY & 0.503 & 0.359 & 0.000 & 0.000 & 0.802 & 0.692 & 0.000 & 0.000 \\
EEA & 0.061 & 0.12 & 0.120 & 0.396 & 0.206 & 0.046 & 0.120 & 0.396 \\
\hline
\end{tabular}

Intra-Class Correlation Coefficient (single and mean ICC) between open (OE) and closed eyes (CE) of the centre of pressure of sway path length (CSPL), Naiperian logarithm of the ellipse of confidence area (LNEA), of the X mean (LNX) and of the $Y$ mean (LNY), eccentricity of the ellipse area (EEA) within time of day. Significant differences was showed with " $p$ " value between open and closed eyes. 


\section{Discussion}

Carrying on a study on a complex and not linear system, as the postural one ${ }^{35,36}$, forces us to make methodological choices in order to avoid bias. The main limit of the study has been the impossibility to fully control the subjects' adherence to the given indications because subjects were volunteers. Stabilometric test was considered an objective and reliable test ${ }^{23,} 37$ and precedent studies investigated the timeof-day effect on postural stability, resuming that: static postural balance in single-foot stance is not affect by a time-of-day effect ${ }^{21}$, postural stability is influenced by time-of-day ${ }^{21}$, random but not significant variations are present during the day ${ }^{23}$. Literature about this issue is not homogeneous, moreover the stabilometric protocols used to test the time-of-day effects are not the same so it is impossible to compare the results and to get a correct information. The present study, compared to the previous ones, has got a larger number of subjects homogeneous in gender, a larger number of test sessions during the day and considered just the closed eyes trials to avoid postural troubles from vision.

It must be highlighted that each data shows high values of standard deviation, especially for CSPL, LNEA and EEA. It can be read as an index of large variability within the subjects. Results from this study can be compared only with the research of Gagey and Weber $^{23}$ because the stabilometric protocol (i.e., duration or feet position) was the same, while the two other studies took into account ${ }^{21,22}$ used different stabilometric protocols (i.e., duration or single leg test) and the results can not be compared. The study confirm the previous results ${ }^{23}$ and no time-of-day effects are present on postural stability with closed/open eyes, so the null hypothesis must be rejected.

It must be highlighted the high standard deviation for some parameters that are usually used in clinical practice for the test interpretation, such as the CSPL. The increasing trend for such parameters in the evening hours may be due to weariness of the postural and nervous system or to the tipical hormonal profile of the evening hours. The center of the Cop trajectory, expressed by the $x$ and $y$ mean coordinates, do not change during the day. This aspect suggests that no time-of-day effect seems to influence the postural strategy used to maintain the equilibrium point of the Cop. The not significant increase during the day of postural oscillations frequencies, confirms the trend of CSPL and LNEA and the possibility that in the evening hours the proprioceptive system does not work at best. Indeed the ICC procedures confirm the reliability and the objectivity of stabilometry ${ }^{23,37}$, but at the same time the small ICC single measurement values must be stressed suggesting a high subject-effect on this postural evaluation. The ICC showed that in the closed/open eyes condition there is a high probability that the ellipse of confidence area is smaller and the postural oscillations are faster, suggesting and augmented energetic expenditure due to the additional work of the proprioceptive system. Da- ta from this study coincides with previous studies both for reliability of stabilometry and for lack of time-of-day effect $^{23}, 37$. The absence of significant variations suggest that there is not a better time range compared to another one to perform a stabilometric evaluation of posture with closed/open eyes, proprioceptive system is not affected by the time-of-day. At the same time individual variations during the day are not statistically significant, but still, they are present: it can be considered, for clinical use, to test the same patient always in the same time range to be sure to correctly read the test results always in the same modalities. The limits of this study, testing only male subject ${ }^{38-41}$, good healthy and age-class, should be overcome with future studies, by testing the open and closed eyes conditions with the same modalities of the present study with new inclusion of criteria (i.e., injury and elderly subjects) ${ }^{42}$.

\section{Acknowledgements}

The authors gratefully thank all athletes who took part in the study and MSc Fabio Sergi, Nicola Ricciardelli, Umberto Pilagatti for supporting this research.

\section{Competing interests}

The authors declare no conflict of interest related to the present manuscript.

\section{Funding}

No funding was received for this article.

\section{Authors contribution}

All authors contributed equally to this manuscript.

\section{References}

1. Vando S, Unim B, Cassarino SA, Padulo J, Masala D. Effectiveness of perceptual training - proprioceptive feedback in a virtual visual diverse group of healthy subjects: a pilot study. Epidemiology Biostatistics and Pubblic Health. 2013;10(2) e8844-1-e8844-10.

2. Vando S, Filingeri D, Maurino L, Chaabene H, Bianco A, Salernitano $\mathrm{G}$, et al. Postural adaptations in preadolescent karate athletes due to a one week karate training cAMP. J Hum Kinet. 2013;38:45-52.

3. Terekhov Y. Stabilometry as a diagnostic tool in clinical medicine. Can Med Assoc J. 1976;115(7):631-633.

4. Terekhov Y. Stabilometry and some aspects of its applications-a review. Biomed Eng. 1976; 11(1):12-15.

5. Pau M, Loi A, Pezzotta MC. Does sensorimotor training improve the static balance of young volleyball players? Sports Biomech. 2012; 11(1):97-107.

6. Lakie M, Caplan N, Loram ID. Human balancing of an inverted pendulum with a compliant linkage: neural control by anticipatory intermittent bias. J Physiol. 2003;551(Pt 1):357-370. 
7. Kantner RM, Rubin AM, Armstrong CW, Cummings V. Stabilometry in balance assessment of dizzy and normal subjects. Am J Otolaryngol. 1991;12(4):196-204.

8. Nardone A, Godi M, Grasso M, Guglielmetti S, Schieppati M. Stabilometry is a predictor of gait performance in chronic hemiparetic stroke patients. Gait Posture. 2009;30(1):5-10.

9. Narita S, Kurose M, Kobayashi K, Himi T. Study on 242 inpatients reporting vertigo and dizziness. Nihon Jibiinkoka Gakkai Kaiho. 2003;106(1):21-27.

10. Oliveira LF, Vieira TM, Macedo AR, Simpson DM, Nadal J. Postural sway changes during pregnancy: a descriptive study using stabilometry. Eur J Obstet Gynecol Reprod Biol. 2009;147(1):25-28.

11. Rogers ME, Rogers NL, Takeshima N, Islam MM. Methods to assess and improve the physical parameters associated with fall risk in older adults. Prev Med. 2003;36(3):255-264.

12. Ammar A, Chtourou H, Trabelsi K, Padulo J, Turki M, El AK, et al. Temporal specificity of training: intra-day effects on biochemical responses and Olympic-Weightlifting performances. J Sports Sci. 2015;33(4):358-368.

13. Atkinson G, Reilly $T$. Circadian variation in sports performance. Sports Med. 1996;21(4):292-312.

14. Honma K. Biological clock and sleep. Brain Nerve. 2012;64(6): 639-646.

15. Padulo J, Ardigò LP. Letter to the Editor concerning "Vertebral rotation in adolescent idiopathic scoliosis calculated by radiograph and back surface analysis-based methods: correlation between the Raimondi method and rasterstereography". Eur Spine J. 2013;22(10):2336-2337.

16. Padulo J, Ardigò LP. Vertebral rotation in adolescent idiopathic scoliosis calculated by radiograph and back surface analysis-based methods: correlation between the Raimondi method and rasterstereography. Eur Spine J. 22:2336-2337: Statistical perspectives part II. Eur Spine J. 2014; 23(4):922-923.

17. Padulo J, Ardigò LP. Formetric rasterstereography: a new perspective. Osteoporos Int. 2014;25(5):1651-1652.

18. Padulo J, Ardigò LP. Evaluating BCl devices: a statistical perspective. Ergonomics. 2014;57(2):282-283.

19. Padulo J, Maffulli N, Ardigò LP. Signal or noise, a statistical perspective. Proc Natl Acad Sci U S A. 2014;111(13):e1160.

20. Padulo J, Ardigò LP. Formetric 4D Rasterstereography. Biomed Res Int. 2014.

21. Gribble PA, Tucker WS, White PA. Time-of-day influences on static and dynamic postural control. J Athl Train. 2007; 42(1):35-41.

22. Forsman P, Haeggstrom E, Wallin A, Toppila E, Pyykko I. Daytime changes in postural stability and repeatability of posturographic measurements. J Occup Environ Med. 2007;49(6): 591-596.

23. Gagey PM, Weber B. Study of intra-subject random variations of stabilometric parameters. Med Biol Eng Comput. 2010; 48(8):833-835.

24. Padulo J, Oliva F, Frizziero A, Maffulli N. Muscles, Ligaments and Tendons Journal. Basic principles and recommendations in clinical and field science research. MLTJ. 2013;4: 250-252.

25. Yagi K. Multivariate statistical analysis in stabilometry in human upright standing (the first report)-age-related factor. $\mathrm{Ni}$ hon Jibiinkoka Gakkai Kaiho. 1989;92(6):899-908.
26. Vieira TM, de Oliveira LF, Nadal J. An overview of age-related changes in postural control during quiet standing tasks using classical and modern stabilometric descriptors. J Electromyogr Kinesiol. 2009;19(6):e513-e519.

27. Mainenti MR, de Oliveira LF, De Melo Tavares De Lima MA, Nadal J. Stabilometric signal analysis in tests with sound stimuli. Exp Brain Res. 2007;181(2):229-236.

28. Romero-Franco N, Martinez-Lopez EJ, Lomas-Vega R, HitaContreras F, Osuna-Perez MC, Martinez-Amat A. Short-term effects of proprioceptive training with unstable platform on athletes' stabilometry. J Strength Cond Res. 2013;27(8):21892197.

29. Kapteyn TS, Bles W, Njiokiktjien CJ, Kodde L, Massen CH, Mol JM. Standardization in platform stabilometry being a part of posturography. Agressologie. 1983;24(7):321-326.

30. Sutow EJ, Maillet WA, Taylor JC, Hall GC. In vivo galvanic currents of intermittently contacting dental amalgam and other metallic restorations. Dent Mater. 2004;20(9):823-831 .

31. Scoppa F, Capra R, Gallamini M, Shiffer R. Clinical stabilometry standardization: basic definitions - acquisition intervalsampling frequency. Gait Posture. 2013;37(2):290-292.

32. Nishiwaki Y, Takebayashi T, Imai A, Yamamoto M, Omae K Difference by instructional set in stabilometry. J Vestib Res. 2000;10(3):157-161.

33. Faul F, Erdfelder E, Lang AG, Buchner A. G*Power 3: a flexible statistical power analysis program for the social, behavioral, and biomedical sciences. Behav Res Methods. 2007;39 (2):175-191.

34. Hopkins WG. Measures of reliability in sports medicine and science. Sports Med. 2000;30(1):1-15.

35. Gagey PM, Martinerie J, Pezard L, Benaim C. Static balance is controlled by a non-linear dynamic system. Ann Otolaryngo Chir Cervicofac. 1998;115(3):161-168.

36. Sasaki O, Usami S, Gagey PM, Martinerie J, Le Van QM, Arranz $P$. Role of visual input in nonlinear postural control system. Exp Brain Res. 2002;147(1):1-7.

37. Ruhe A, Fejer R, Walker B. The test-retest reliability of centre of pressure measures in bipedal static task conditions-a systematic review of the literature. Gait Posture. 2010;32(4):436445.

38. Attene G, Pizzolato F, Calcagno G, Ibba G, Pinna M, Salernitano $\mathrm{G}$, et al. Sprint vs. intermittent training in young female basketball players. J Sports Med Phys Fitness. 2014;54(2): 154-161.

39. Padulo J, Di Giminiani R, Ibba G, Zarrouk N, Moalla W, Attene $\mathrm{G}$, et al. The acute effect of whole body vibration on repeated shuttle-running in young soccer players. Int J Sports Med. 2014;35(1):49-54.

40. Padulo J, Filingeri D, Chamari K, Migliaccio GM, Calcagno G, Bosco G, et al. Acute effects of whole-body vibration on running gait in marathon runners. J Sports Sci. 2014;32(12):11201126.

41. Laffaye G, Collin JM, Levernier G, Padulo J. Upper-limb Power Test in Rock-climbing. Int J Sports Med. 2014;35(8):670675.

42. Haddad M, Padulo J, Chamari K. The Usefulness of SessionRPE Method for Training Load Monitoring Despite Several Influences on Perceived Exertion. Int J Sports Physiol Perform. 2014;9(9):882-883. 